\section{A FORTRAN IV program for the Stuart-Maxwell test}

\section{WILLIAM P. DUNLAP \\ Tulane University, New Orleans, Louisiana}

When categorical measures are taken on independent groups, the statistical procedure of choice is the familiar Pearson chi-square test. The Pearson chi-square should be used only when the categorical data are independent, meaning that each subject is counted once and only once, and each subject's score is independent of each other subject's score. There are two commonly used experimental designs that produce nonindependent categorical data: designs in which the subjects in the different groups have been matched in some way, and designs in which the subjects are measured more than once, for example, a prepost design in which the subject is measured before and after a treatment. Unfortunately, with the latter, a somewhat bewildering number of statistical procedures are available, depending upon the type of categorical data and the number of repeated measures involved. If the data were continuous rather than categorical, one would ordinarily use a repeated measures analysis of variance. If the data were such that the magnitude of change from pretest to posttest could be ranked, the Wilcoxon signedrank test would be appropriate. If only the direction of change from pre to post could be measured, then the sign test or McNemar's test for change could be used.

Generalizations of these tests to more complex situations include: Friedman's test, which applies to three or more trials, and for which the subject's data across trials must be ordered or ranked; and Cochran's test, for which the data across repeated measures are either 0 s or $1 \mathrm{~s}$, indicating that an event or state either did or did not occur. A third generalization of the sign test or McNemar's test is the Stuart-Maxwell test, the topic of the present paper.

The Stuart-Maxwell Test. The Stuart-Maxwell test (Stuart, 1955; Maxwell, 1970) is appropriate when there are either two measures on the same subjects or two matched groups of subjects and the data are categorical. If the data have two categories only, the Stuart-Maxwell test is identical to McNemar's test. When there are three data categories (see, e.g., Table 1), the Stuart-Maxwell test can be solved by a direct algebraic expression (Fleiss \& Everitt, 1971). When there are more than three data categories, the procedure requires matrix inversion, which is the reason the program described herein was written.

The data for the Stuart-Maxwell test are usually written in the form of a contingency table. For example (see Table 1), we ask 78 subjects for which candidate they intend to vote before some important event (such as a con-

The author's mailing address is: Department of Psychology, Tulane University, New Orleans, LA 70118.
Table 1

Example of a $3 \times 3$ Experiment on Voting Intentions Before and After a Hypothetical Event

\begin{tabular}{lcccc}
\hline & \multicolumn{3}{c}{ After } & \\
\cline { 2 - 4 } \multicolumn{1}{c}{ Before } & Republican & Democrat & Undecided & Row Total \\
\hline Republican & 20 & 10 & 2 & 32 \\
Democrat & 3 & 25 & 1 & 29 \\
Undecided & 2 & 5 & 10 & 17 \\
Column Total & 25 & 40 & 13 & 78 \\
\hline
\end{tabular}

Note-Stuart-Maxwell chi-square on $2 d f=6.4026, p=.04071$.

vention, an international crisis, etc.), and then again after the event. These data suggest that, before the event, the Republican candidate is favored and there are a number of undecided votes. After the event, the Democrat is favored, and there are fewer undecided votes. If the standard Pearson's chi-square test is applied to such data, it tests only the hypothesis of independence; that is, is the tendency to vote in a particular way after the event related to the intended vote before the event? Clearly, this hypothesis is of little interest because we expect repeated measures on the same subjects to be nonindependent. The Stuart-Maxwell procedure, on the other hand, specifically tests whether row marginal frequencies differ significantly from the corresponding column marginal frequencies. In the example, the 32 Republican supporters before the event are compared with the 25 Republican voters after the event, the 29 Democratic voters before with the 40 after, and the 17 undecided pre- with the 13 postevent. Notice that the difference between the 32 Republican voters before and the 25 after is 7 , which is the difference between the 12 voters who moved out of the Republican category (10 to the Democrats and 2 to undecided) and the 5 who moved into the Republican category ( 3 former Democratic supporters and 2 undecided). Therefore, the Stuart-Maxwell test is a test for change in categories from before to after the event, not a test for independence.

The statistic produced by the Stuart-Maxwell test is a chi-square with degrees of freedom equal to the number of data categories minus one. For the example in Table 1 , chi-square on two degrees of freedom $=6.4026(\mathrm{p}=$ .04071 ); thus, the change in voting tendency due to the event was significant for this artificial example. For other examples that can be used to check proper program functioning, see Fleiss (1973, pp. 78-79) or Stuart (1955, pp. 415-416).

McNemar's Test. For the case in which there are only two data categories, as mentioned earlier, the StuartMaxwell test gives the same chi-square value as $\mathrm{McNe}$ mar's test for change. The reader is cautioned, however, that in this case an exact probability can be computed using the binomial distribution, rather than using the chisquare approximate test. The exact test is done by ignor- 
ing all individuals who do not change categories from before the event to after the event. Instead, the number of subjects that change from Category 1 to Category 2 is compared with the number of subjects who change from Category 2 to Category 1. Under the null hypothesis, these two numbers should be equal; that is, the probability of a change in either direction should be 0.5 . The probability associated with the difference between these two frequencies can be computed directly by the application of the binomial distribution with expected proportions of 0.5. (See Dunlap, Myers, \& Silver, 1984, for a program to do the computation.)

The Program. The user is queried interactively for the number of rows (equal to the number of columns) of the square data matrix. Next, the data are entered cell by cell interactively. The program responds with the appropriate chi-square value and degrees of freedom. A subroutine, MATINV, published by Cooley and Lohnes (1971), is used for matrix inversion; however, any matrix inversion subroutine that replaces a matrix by its inverse can be used. Also, if the functions ZPRB and CPRB, described by Dunlap and Duffy (1975), are added, chisquare probabilities may be computed and printed.

Requirements. The program is written in singleprecision FORTRAN IV and runs on a DEC-20 computer. Because the program is interactive, the input and output are through the computer terminal. Several nonstandard FORTRAN statements are used to make the program run efficiently on the DEC system. Users with other systems may have to modify the assignment of logical units, READ statements with an "*" in place of the usual format statement number (this causes free format on the DEC), and format statements that end with the symbol " $\$$," which causes the cursor to remain at the end of the line on the DEC.

Program Availability. A listing of the program may be obtained at no charge from William P. Dunlap, Department of Psychology, Tulane University, New Orleans, Louisiana 70118.

\section{REFERENCES}

COOLEy, W. W., \&OHNES, P. R. (1971). Multivariate data analysis. New York: Wiley.

DUNLAP, W. P., DUFFY, J. A. (1975). FORTRAN IV functions for calculating exact probabilities associated with $\mathrm{z}, \chi^{2}, \mathrm{t}$, and $\mathrm{F}$ values. Behavior Research Methods \& Instrumentation, 7, 59-60.

DunlaP, W. P., Myers, L., \& Silver, N. C. (1984). Exact multinomial probabilities for one-way contingency tables. Behavior Research Methods, Instruments, \& Computers, 16, 54-56.

FLEISS, J. L. (1973). Statistical methods for rates and proportions. New York: Wiley.

FLEISS, J. L., \& EveriTt, B. S. (1971). Comparing the marginal totals of square contingency tables. British Journal of Mathematical and Statistical Psychology, 24, 117-123.

Maxwell, A. E. (1970). Comparing the classification of subjects by two independent judges. British Journal of Psychiatry, 116, 651-655.

StuarT, A. (1955). A test for the homogeneity of the marginal distributions in a two-way classification. Biometrika, 42, 412-416.
Appendix

FORTRAN Program for the Stuart-Maxwell Test

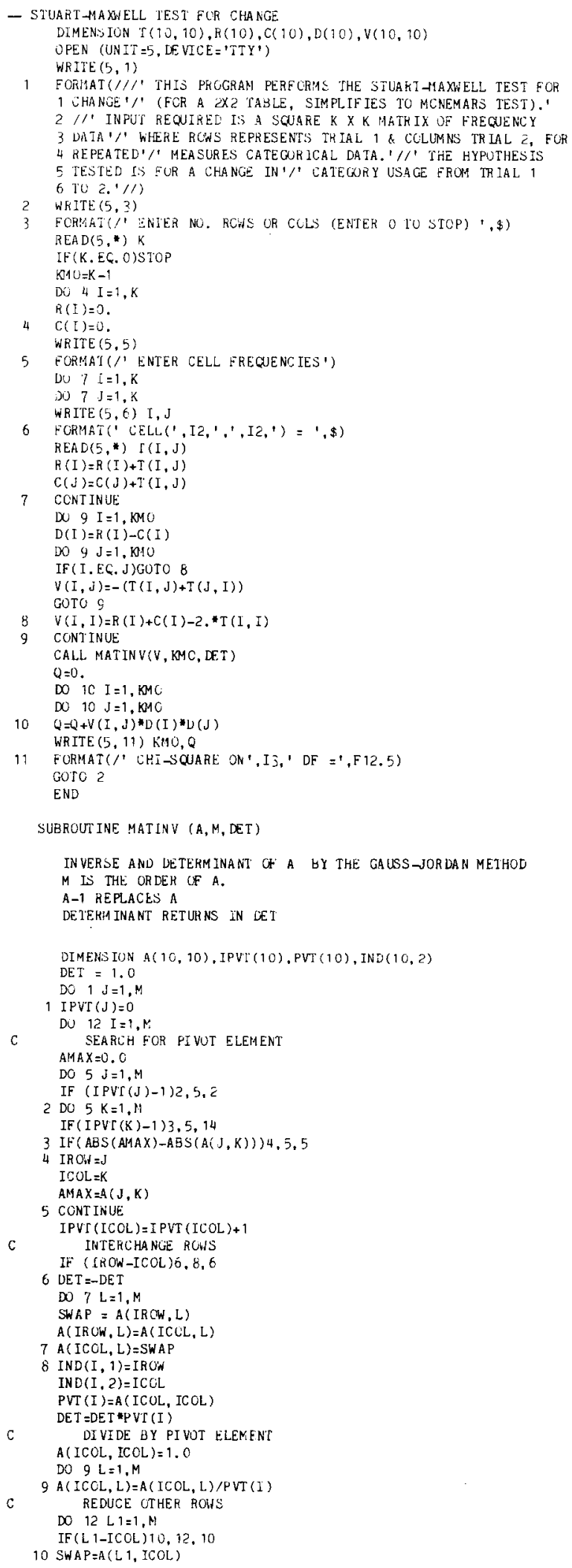


$A(L 1, I C O L)=0.0$

DO $11 L=1, M$

$11 A(L 1, L)=A(L 1, L)-A(I C C L, L)=S W A . P$

12 CONT INUSE

IN'TERCHANGE CCLUMAS

Do $14 \mathrm{I}=1$,

$\operatorname{IF}(\operatorname{IND}(L, 1)-\operatorname{IND}(L, 2)) 13,14,13$

IROW $=$ IND $(L, 1)$

ICCL $=I N D(L, 2)$

DO $14 \mathrm{~K}=1, \mathrm{M}$
SW $A P=A(K, I R$ OW $)$

$A(K, I R O W)=A(K, I C O L)$

$A(K, I C O L)=S W A P$

14 CONTINUE

RETURN

END 